\title{
V8 induces apoptosis and the endoplasmic reticulum stress response in human multiple myeloma RPMI 8226 cells via the PERK-eIF2 $\alpha$-ATF4 signaling pathway
}

\author{
YAPING ZHONG ${ }^{1}$, YONGGANG ZHANG ${ }^{2}$, PING WANG $^{1}$, HONGXIU GAO $^{1}$, CHUNLING XU $^{1}$ and HUI LI ${ }^{1}$ \\ ${ }^{1}$ Department of Hematology; ${ }^{2}$ Deanery, Zhumadian Central Hospital, Zhumadian, Henan 463000, P.R. China
}

Received April 28, 2015; Accepted June 2, 2016

DOI: $10.3892 / 01.2016 .5005$

\begin{abstract}
Multiple myeloma (MM) is a fatal hematological cancer characterized by clonal plasma cell proliferation in the bone marrow. MM has an increasing global incidence and a poor prognosis. There are limited treatment options available for MM, and this is further compounded by the development of drug resistance. The present study demonstrated that 7- $\{4-[\mathrm{Bis}-$ (2-hydroxyethyl)-amino]-butoxy\}-5-hydroxy-8-methoxy-2phenylchromen-4-one (V8), a novel synthetic flavonoid, induced apoptosis in human MM RPMI 8226 cells in a dose- and time-dependent manner, using cell viability assays and flow cytometry. Subsequently, V8-induced apoptosis in RPMI 8226 cells was revealed to occur via mitochondria-mediated pathways. The activity of caspase- $3,-8$ and -9 , and the mRNA level of B-cell lymphoma 2 (Bcl-2) and B-cell lymphoma-extra large were greatly increased, while the expression of Bcl-2-like protein 4 and $\mathrm{BH} 3$ interacting domain death agonist was significantly decreased in RPMI 8226 cells following V8 treatment, as observed using quantitative polymerase chain reaction (qPCR). In addition, western blotting revealed that the release of mitochondrial cytochrome $c$ into the cytosol was promoted by V8. Furthermore, a clear alteration in endoplasmic reticulum (ER) stress was observed in cells treated with V8; upregulation of glucose-regulated protein (GRP) 78, GRP94, C/EBP homologous protein, cleavage of caspase-12, phosphorylated protein kinase RNA-like endoplasmic reticulum kinase (p-PERK), phosphorylated eukaryotic initiation factor $2 \alpha$ (p-eIF2 $\alpha$ ) and activating transcription factor 4 (ATF4) was observed with qPCR and western blotting, suggesting that V8-induced apoptosis is involved in the ER stress response. Overall, the present results demonstrated that V8 induced apoptosis in human MM RPMI 8226 cells via the PERK-eIF2 $\alpha$-ATF4 ER stress response pathway, which may provide novel directions for exploiting this compound as a potential anti-neoplastic drug for MM therapy.
\end{abstract}

Correspondence to: Professor Yaping Zhong, Department of Hematology, Zhumadian Central Hospital, 747 Zhonghua Road, Zhumadian, Henan 463000, P.R. China

E-mail: zhongyapinghn@163.com

Key words: V8, apoptosis, endoplasmic reticulum stress, myeloma

\section{Introduction}

Multiple myeloma (MM) is a fatal hematological cancer, which is characterized by clonal plasma cell proliferation in the bone marrow, presence of osteolytic bone destruction causing severe bone pain, pathological fractures and hypercalcaemia (1). The global incidence of MM has increased continuously in the last decade (2). Despite overall survival rates improving significantly with recent therapeutic advancements, including autologous stem cell transplantation, proteasome inhibitors and immunomodulatory drugs, MM remains an incurable disease with a median survival time of 4-5 years in adults, due to its resistance to chemotherapeutic drugs $(3,4)$. Therefore, the development of novel alternative approaches to overcome drug resistance and improve outcomes in MM is urgently required.

Flavonoids are widely distributed in plants and possess a wide range of biological and pharmacological activities, including anti-allergic, anti-inflammatory, antioxidant, anti-microbial and anti-diarrheal activities $(5,6)$. Previous studies in vitro and in vivo have revealed that flavonoids have anti-cancer effects in various types of cancer, including human breast, lung and colorectal cancer, hepatocellular carcinoma, osteosarcoma and glioma, by inducing apoptosis, increasing chemotherapy sensitivity and suppressing metastasis (7-9).

Apoptosis is the major mechanism for cancer cell elimination. Previous studies have demonstrated that flavonoids trigger apoptosis in numerous types of human cancer cells through endoplasmic reticulum (ER) stress-dependent apoptotic pathways and mitochondrial-mediated apoptotic pathways (10-12). A recent study revealed that 7-\{4-[Bis-(2-hydroxyethyl)-amino]-butoxy\} -5-hydroxy-8-methoxy-2-phenylchromen-4-one (V8), a novel flavonoid that is synthesized from the natural product wogonin in two steps (Fig. 1A), induces apoptosis in hepatocellular carcinoma cells through the reactive oxygen species-mediated ER stress pathway (13). However, to the best of our knowledge, no study has systematically investigated the cytotoxic effects and mechanisms of V8 on MM cells. Therefore, the present study investigated the cytotoxic potential of V8, and the mechanism by which it acts, on MM cells by examining cell viability and apoptosis signals in human MM RPMI 8226 cells. The present study provides detailed information concerning the cytotoxic effects of V8 on MM cells and offers a basic foundation for the clarification of its toxicity mechanisms. 


\section{Materials and methods}

Reagents and antibodies. V8 [purity, >99.5\%; obtained from Aiqing He, Nantong University, Jiangsu, China (14)] was diluted in dimethyl sulfoxide (DMSO) to $0.1 \mathrm{M}$ and stored at $-20^{\circ} \mathrm{C}$. Cell counting kit-8 (CCK-8; catalog no. CK04-3000T) was purchased from Dojindo Molecular Technologies, Inc. (Kumamoto, Japan). FITC Annexin V Apoptosis Detection kit (catalog no. 556570) was purchased from BD Biosciences (Franklin Lakes, NJ, USA). Mouse monoclonal $\beta$-tubulin (catalog no. sc-5274; 1:1,000 dilution) and mouse monoclonal $\beta$-actin (catalog no. sc-47778; 1:1,000 dilution) primary antibodies were purchased from Santa Cruz Biotechnology, Inc. (Dallas, TX, USA). Mouse monoclonal cytochrome $c$ oxidase subunit IV (COX4; catalog no. 4844; 1:1,000 dilution), rabbit monoclonal cleaved caspase-12 (catalog no. 2202; 1:500 dilution), rabbit monoclonal phosphorylated protein kinase RNA-like endoplasmic reticulum kinase (p-PERK; catalog no. 4370S; 1:1,000 dilution), rabbit monoclonal phosphorylated eukaryotic initiation factor $2 \alpha$ (p-eIF2 $\alpha$; catalog no. 3597; 1:1,000 dilution) and rabbit monoclonal activating transcription factor 4 (ATF4; catalog no. 11815; 1:1,000 dilution) antibodies were obtained from Cell Signaling Technology, Inc. (Danvers, MA, USA). Mouse monoclonal cytochrome $c$ (cyto $c$; catalog no. 13560; 1:800 dilution) antibody was purchased from Santa Cruz Biotechnology, Inc. Goat anti-mouse IgG-conjugated horseradish peroxidase (catalog no. sc-2004; 1:20,000 dilution) and CY3-conjugated (catalog no. sc-2020; 1:5,000 dilution) secondary antibodies were obtained from Santa Cruz Biotechnology, Inc.

Cell culture and cell viability. Human MM RPMI 8226 cell line was purchased from the Chinese Academy of Sciences (Beijing, China) and maintained in RPMI-1640 (Thermo Fisher Scientific, Inc., Waltham, MA, USA) supplemented with $10 \%$ fetal bovine serum (Gibco ${ }^{\circledR}$; Thermo Fisher Scientific, Inc.), $2 \mathrm{mM}$ glutamine and $100 \mathrm{U} / \mathrm{ml}$ penicillin-streptomycin (Sigma-Aldrich) at $37^{\circ} \mathrm{C}$ in $5 \% \mathrm{CO}_{2}$. Following treatment with various concentrations of $\mathrm{V} 8(0,25,50,75$ and $100 \mu \mathrm{M})$, cell viability was measured by CCK-8. The absorbance was measured at $450 \mathrm{~nm}$ using a microplate reader (Bio-Tek Instruments, Inc., Winooski, VT, USA).

Western blotting. Following treatment with various concentrations of V8 $(0,25,50,75$ and $100 \mu \mathrm{M})$, RPMI 8226 cells were harvested and washed with ice-cold phosphate-buffered saline (PBS). Cell lysates were prepared using radioimmunoprecipitation assay (RIPA) buffer (Cell Signaling Technology, Inc.). The samples were separated by sodium dodecyl sulfate-polyacrylamide gel electrophoresis using a Mini Protean system (Bio-Rad Laboratories, Inc., Hercules, CA, USA) on a 10\% gel and transferred to a nitrocellulose membrane (EMD Millipore, Billerica, MA, USA). Following blocking with 5\% skimmed milk at room temperature for $2.5 \mathrm{~h}$, the membranes were incubated with primary antibodies against cleaved caspase12 , cyto $c, \operatorname{COX} 4, \beta$-actin and $\beta$-tubulin at $4^{\circ} \mathrm{C}$ overnight. The samples were visualized using goat anti-mouse IgG-conjugated horseradish peroxidase antibody at room temperature for 2-3 h, with enhancement from Pierce ${ }^{\mathrm{TM}}$ ECL Western Blotting Substrate (Thermo Fisher Scientific, Inc.). Image J (1.49v;
National Institutes of Health, Bethesda, MD, USA) was used for western blotting analysis.

Flow cytometric analysis of apoptosis. RPMI 8226 cells were cultured in 6-well plates for $48 \mathrm{~h}$ in the presence of $100 \mu \mathrm{M} \mathrm{V8}$. The cells were washed twice with cold PBS and then resuspended in $1 \mathrm{X}$ Binding Buffer at a concentration of $1 \times 10^{6}$ cells $/ \mathrm{ml}$. In total, $100 \mu 1$ of the solution ( $1 \times 10^{5}$ cells) was transferred to a $5 \mathrm{ml}$ culture tube and $5 \mu \mathrm{l}$ FITC Annexin V and $5 \mu \mathrm{l}$ propidum iodide (PI) were added. The cells were gently agitated and incubated for $15 \mathrm{~min}$ at room temperature $\left(25^{\circ} \mathrm{C}\right)$ in the dark. Following incubation, the stained cells were diluted by addition of $400 \mu 1 \mathrm{lX}$ Binding Buffer. Fluorescence was detected using FACSCalibur ${ }^{\mathrm{TM}}$ Flow Cytometer (BD Biosciences) within $1 \mathrm{~h}$ and analyzed by FlowJo flow cytometric data analysis software (FlowJo7.6, LLC, Ashland, OR, USA). PI and FITC Annexin V positively stained cells were considered to be apoptotic.

RNA isolation and quantification of transcript levels. RNA was isolated from cells treated with $0,25,50,75$ and $100 \mu \mathrm{M}$ V8 using TRIzol reagent (Invitrogen ${ }^{\mathrm{TM}}$; Thermo Fisher Scientific, Inc.). Total RNA was converted to cDNA for quantitative polymerase chain reaction (qPCR) using a High Capacity cDNA Reverse Transcription kit (Applied Biosystems ${ }^{\mathrm{TM}}$; Thermo Fisher Scientific, Inc.), according to the manufacturers protocol. DNase I (catalog no. 18047019; 1:800 dilution; Invitrogen; Thermo Fisher Scientific, Inc.) was used. qPCR was performed to detect the expression of B-cell lymphoma 2 (Bcl-2), Bcl-2-like protein 4 (Bax), BH3 interacting domain death agonist (Bid), B-cell lymphoma-extra large (Bcl-XL), glucose-regulated protein (GRP) 78, GRP94 and C/EBP homologous protein (CHOP) using the SYBR Green PCR MasterMix and an ABI 7500 Real-time PCR System (Applied Biosystems $^{\mathrm{TM}}$; Thermo Fisher Scientific, Inc.). The primers used in the qPCR were synthesized by Sangon Biotech (Shanghai, China). The cycling conditions were as follows: Stage 1 , hold at $95^{\circ} \mathrm{C}$ for $120 \mathrm{sec}$; stage $2,95^{\circ} \mathrm{C}$ for $15 \mathrm{sec}$ and $60^{\circ} \mathrm{C}$ for $35 \mathrm{sec}$, for 40 cycles; and stage 3 , dissociation. Relative mRNA expression was determined by the $2^{-\Delta \Delta C q}$ method (15) vs. glyceraldehyde 3-phosphate dehydrogenase. Sequences for PCR primers are listed in Table I. The experiments were repeated three times.

Caspase-3, - 8 and -9 activity assay. Activation of caspase- $3,-8$ and -9 was measured using the Fluorometric Assay kit (Calbiochem $^{\circledR}$; EMD Millipore), according to the manufacturer's protocol. In brief, RPMI 8226 cells were treated with various concentrations of $\mathrm{V} 8(0,25,50,75$ and $100 \mu \mathrm{M})$ for $24 \mathrm{~h}$. The treated cells were harvested and washed with ice-cold PBS. Cell lysates were obtained by adding $100 \mu 1$ RIPA buffer (Cell Signaling Technology, Inc.) for $1 \times 10^{5}$ cells. Subsequently, caspase inhibitors (caspase-3, DEVD-CHO; caspase-8, z-IETD-FMK; caspase-9, z-LEHD-FMK) were added to the cell lysates and incubated for $30 \mathrm{~min}$. Reaction buffer and fluorogenic peptide substrates $(10 \mu \mathrm{l})$ (caspase-3, Ac-DEVD-A MC; caspase-8, Ac-IETD-A MC; caspase-9, Ac-LEHD-A MC) were added to the cell lysates, and incubated at $37^{\circ} \mathrm{C}$ in the dark for $2 \mathrm{~h}$. Lysate from RPMI 8226 cells treated with DMSO was used as a control group. The activation of 
Table I. Primer sequences used in quantitative polymerase chain reaction.

Bcl-2-like protein 4

$\mathrm{BH} 3$ interacting domain death agonist

Bcl-2

Bcl-extra large

GRP78

GRP94

C/EBP homologous protein

Glyceraldehyde 3-phosphate dehydrogenase

TTTTGCTTCAGGGTTTCATC
GGTCAACAACGGTTCCAG
CTGGGAGAACAGGGTACGATAA
TGTGCGTGGAAAGCGTAG
CTCTGCCTCACCTCGCTCCA
CCACCTTCATCATCTACC
ACCAGGAAACGGAAACAG
AAGGTCATCCCTGAGCTGAA

GACACTCGCTCAGCTTCTTG CATCGTAGCCCTCCCACT GGCTGGGAGGAGAAGATGC AGTGAGCCCAGCAGAACC TCGCAATAGCAATGCCAATC ATGAGCCCTAACAGCAC TCACCATTCGGTCAATCA TGCTGTAGCCAAATTCGTTG

Bcl, B-cell lymphoma; GRP, glucose-regulating protein.

A

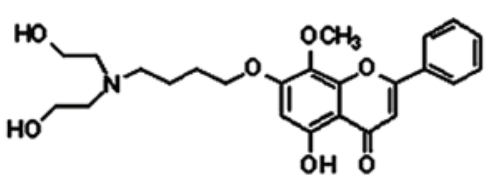

D

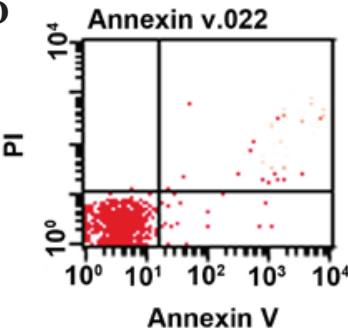

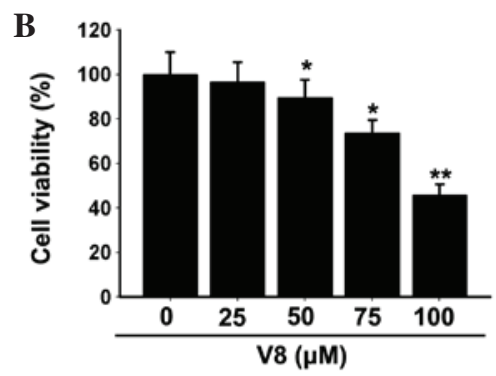
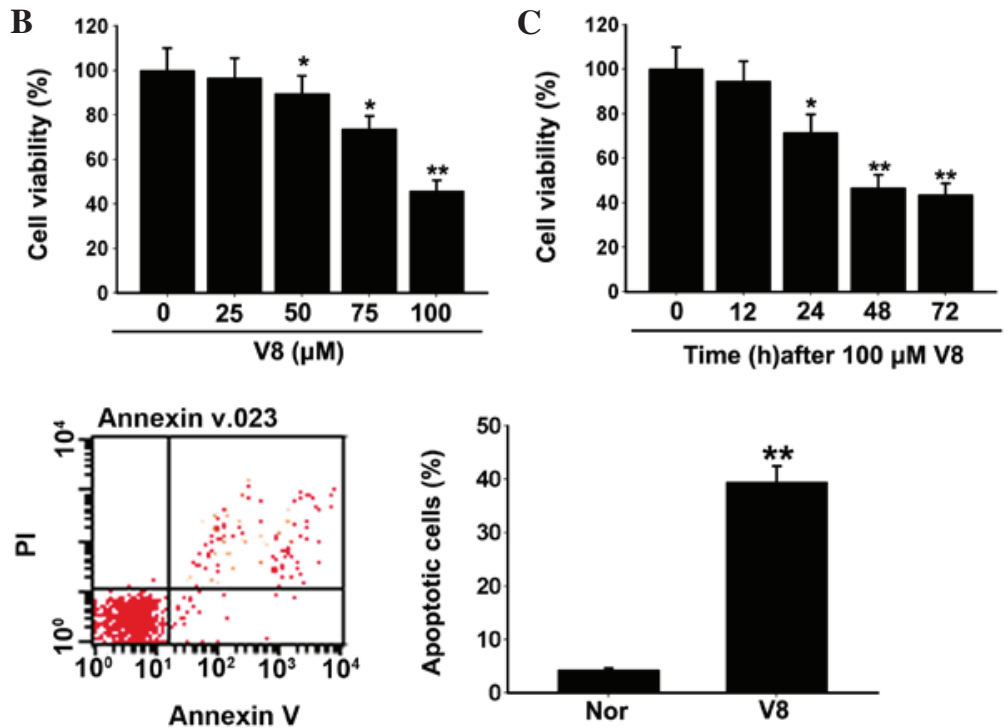

Figure 1. Effects of V8 on cell viability and apoptosis of human multiple myeloma RPMI 8226 cells. (A) Molecular structure of V8. (B and C) Cell viability of RPMI 8226 cells following exposure to (B) various concentrations of V8 and (C) V8 at $100 \mu \mathrm{M}$ for various periods of time, as determined by cell counting kit- 8 assay. (D) Apoptosis of control cells (left) and RPMI 8226 cells (right) following treatment with $100 \mu \mathrm{M}$ V8 for $48 \mathrm{~h}$ was detected by Annexin V-fluorescein isothiocyanate/PI double staining. Data are presented as the mean \pm standard error of the mean of three independent experiments. ${ }^{*} \mathrm{P}<0.05$, ${ }^{* *} \mathrm{P}<0.01$ vs. control. V8, 7-\{4-[Bis-(2-hydroxyethyl)-amino]-butoxy\}-5-hydroxy-8-methoxy-2-phenylchromen-4-one; PI, propidium iodide; nor, untreated RPMI 8226 cells.

caspases in V8-treated RPMI 8226 cells was measured using the Infinite ${ }^{\circledR} 200$ PRO microplate reader (Tecan Group Ltd, Männedorf, Switzerland) at a wavelength of $405 \mathrm{~nm}$.

Preparation of cytosolic extracts and mitochondria isolation. Mitochondria Isolation Kit for Cultured Cells (Thermo Fisher Scientific, Inc.) was used for cyto $c$ analysis, according to the manufacturer's protocol. Briefly, RPMI 8226 cells $\left(2 \times 10^{7}\right)$ treated with $0,25,50,75$ and $100 \mu \mathrm{M}$ V8 were washed with ice-cold PBS and resuspended in $800 \mu \mathrm{l}$ reagent A. Following incubation on ice for $2 \mathrm{~min}, 10 \mu \mathrm{l}$ reagent $\mathrm{B}$ was added and incubated on the ice for $5 \mathrm{~min}$ and vortexed every minute. Subsequently, $800 \mu \mathrm{l}$ reagent $\mathrm{C}$ was added and centrifuged in a microcentrifuge at $700 \mathrm{x} \mathrm{g}$ for $10 \mathrm{~min}$ at $4^{\circ} \mathrm{C}$ to collect the supernatant. The supernatant was transferred to a new tube and subjected to centrifugation at $12,000 \mathrm{x}$ g for $15 \mathrm{~min}$ at $4^{\circ} \mathrm{C}$. The supernatant was collected as cytosolic extracts and the mitochondria were washed with reagent $\mathrm{C}$ and centrifuged at $12,000 \mathrm{x} \mathrm{g}$ for $10 \mathrm{~min}$ at $4^{\circ} \mathrm{C}$ for the analysis of cyto $c$.
Distribution of cyto $c$ in cytosolic extracts and isolated mitochondria was determined by western blotting and normalized to $\beta$-tubulin and COX4, respectively.

Immunofluorescent staining. RPMI 8226 cells were plated onto coverslips the day prior to treatment with V8. Following exposure to $75 \mu \mathrm{M} \mathrm{V8}$ for $48 \mathrm{~h}$, the cells were fixed with $4 \%$ paraformaldehyde for $30 \mathrm{~min}$, permeabilized with PBS with Tween $20(0.5 \%)$ for $10 \mathrm{~min}$ and incubated with primary cleaved caspase-12 antibodies at $4^{\circ} \mathrm{C}$ overnight, followed by CY3-conjugated secondary antibody staining at room temperature for $2 \mathrm{~h}$. Following washing, the cells were stained with 4',6-diamidino-2-phenylindole (1 mmol/1; Sigma-Aldrich) for $5 \mathrm{~min}$. The cells were observed using a fluorescence microscope (Carl Zeiss AG, Oberkochen, Germany).

Statistical analysis. All data are expressed as the mean \pm standard error of the mean from 3 or 4 independent experiments and analyzed using Student's t-test. Statistical analysis was 

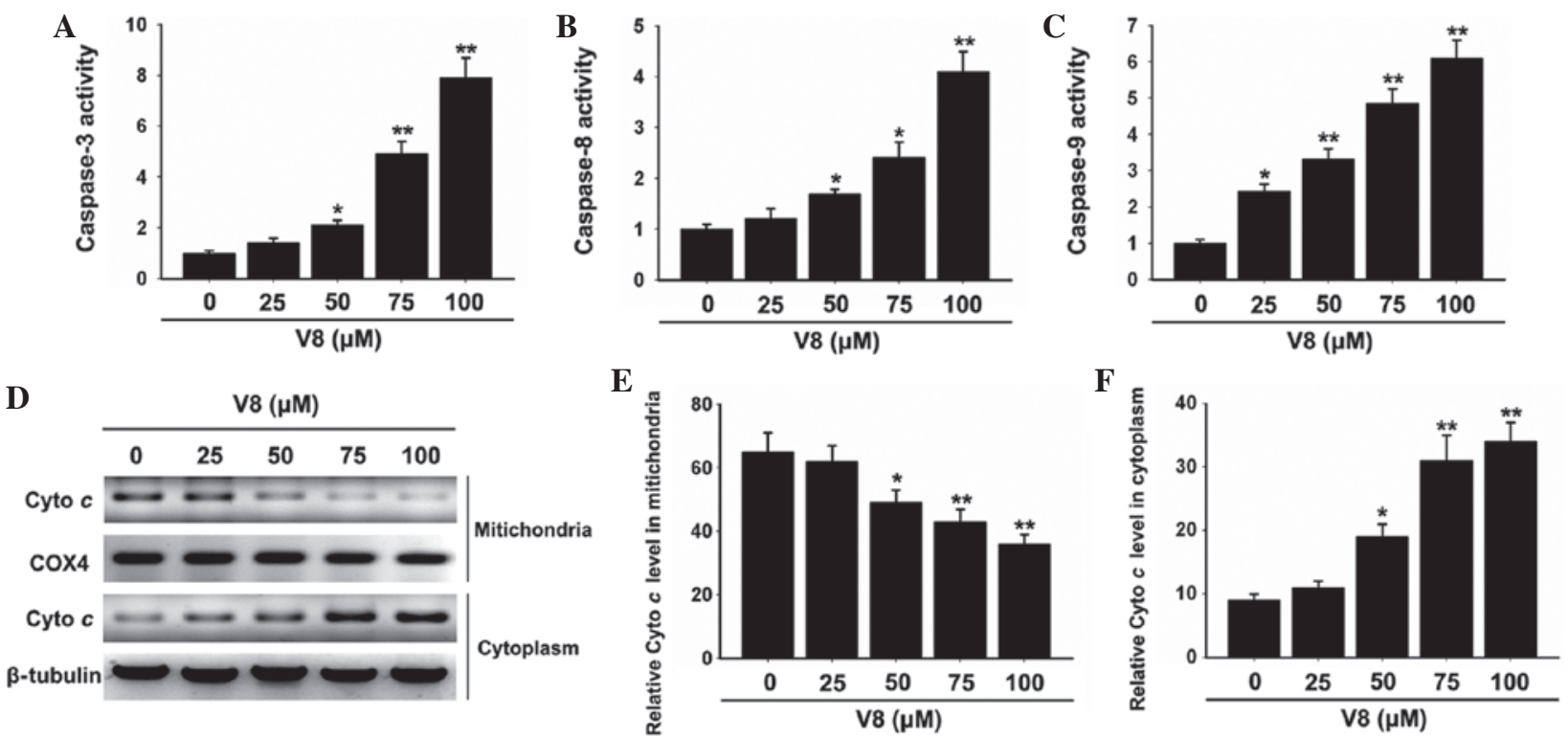

Figure 2. Caspases activities and cyto $c$ release following exposure to V8. (A-C) Human multiple myeloma RPMI 8226 cells were treated with various concentrations of V8 for $48 \mathrm{~h}$ and the activation of caspase-3, -8 and -9 was detected by fluorometric assay. (D) Western blotting was performed to determine the level of cyto $c$ in the mitochondria and cytoplasm of RPMI 8226 cells. (E and F) Densitometry showing the ratio of cyto $c$ relative to (E) COX4 in mitochondria and (F) $\beta$-tubulin in the cytoplasm for each V8 concentration. Data are presented as the mean \pm standard error of the mean of three independent experiments. ${ }^{*} \mathrm{P}<0.05,{ }^{* *} \mathrm{P}<0.01$ vs. untreated cells. V8, 7-\{4-[Bis-(2-hydroxyethyl)-amino]-butoxy $\}$-5-hydroxy-8-methoxy-2-phenylchromen-4-one; cyto $c$, cytochrome $c$; COX4, cytochrome $c$ oxidase subunit IV.

performed using SigmaPlot software version 10.0 (Systat Software, Inc., San Jose, CA, USA). P $<0.05$ was considered to indicate a statistically significant difference.

\section{Results}

$V 8$ induces apoptosis of RPMI 8226 cells. To investigate the effect of V8 on human MM cell growth, RPMI 8226 cells were treated with various concentrations of V8 $(0,25,50,75$ and $100 \mu \mathrm{M})$ and cell viability and apoptosis were evaluated. Inhibition of cell viability was observed following treatment with V8 in a dose- and time-dependent manner (Fig. 1B and $\mathrm{C} ; \mathrm{P}<0.05$ ). Following a $48 \mathrm{~h}$ exposure to V8 at $100 \mu \mathrm{M}$, RPMI 8226 cells exhibited typical apoptotic alterations, including cell shrinkage and loss of normal nuclear architecture (date not shown). To elucidate these observations more definitively, FITC Annexin-V/PI staining was performed. The percentage of Annexin-V labeled apoptotic cells was significantly upregulated following treatment with V8 compared with the control (Fig. 1D; P<0.05).

Caspase pathway was activated in RPMI 8226 cells following treatment with $V 8$. To determine the possible mechanism of action in which V8 induces apoptosis of RPMI 8226 cells, alterations in the expression of critical apoptosis-associated factors were evaluated. Caspase protease activation was assessed to determine the involvement in the cell death response. Notably, the activity of caspase- $-3,-8$ and -9 was clearly elevated in cells treated with V8 (Fig. 2A-C; P<0.05). Subsequently, the effects of $\mathrm{V} 8$ on the release of mitochondrial cyto $c$ into the cytosol of cells was evaluated. Western blot analysis revealed that the level of cyto $c$ was decreased in mitochondria and increased in the cytoplasm with increasing concentrations of $\mathrm{V} 8$, indicating that $\mathrm{V} 8$ promotes the release of mitochondrial cyto $c$ into the cytosol (Fig. 2D-F; P<0.05).
Expression of apoptosis factors was altered following V8 treatment. To further investigate the molecular basis of the apoptosis induced by V8 in RPMI 8226 cells, the expression of apoptosis-associated factors were evaluated. The Bcl-2 family, also known as fundamental death regulatory proteins, are key regulators in mitochondrial outer membrane permeabilization (16). As shown in Fig. 3, the mRNA expression of Bax and Bid was clearly downregulated $(\mathrm{P}<0.05)$, while the mRNA expression of Bcl-2 and Bcl-XL was significantly upregulated $(\mathrm{P}<0.05)$ following treatment with 50,75 and $100 \mu \mathrm{M}$ V8 for $48 \mathrm{~h}$. There was no alteration in the expression of Bax, Bid, $\mathrm{Bcl}-2$ and $\mathrm{Bcl}-\mathrm{XL}$ with low concentrations of V8 $(\mathrm{P}>0.05)$. The alterations observed in Bcl-2 family member expression was consistent with the cellular apoptosis induced by V8.

ER stress response was activated in RPMI 8226 cells following treatment with V8. ER stress has emerged as a key instigator of the intrinsic apoptotic pathway (17). To investigate whether the apoptosis of RPMI 8226 cells, induced by V8, was associated with ER stress, components of the ER stress pathway were evaluated. Notably, a significant upregulation in the ER stress response elements GRP78, GPR94 and CHOP was observed following treatment with 50, 75 and $100 \mu \mathrm{M}$ V8 (Fig. 4A-C; $\mathrm{P}<0.05)$. In addition, cleaved caspase- 12 was significantly increased, as shown by western blotting (Fig. 4D; P<0.05) and immunofluorescence (Fig. 4D and E).

The unfolded protein response (UPR) is activated to prevent further translational loading of the ER in response to ER stress. PERK activates itself by oligomerization and autophosphorylation of the free luminal domain and causes translational attenuation by directly phosphorylating eIF $2 \alpha$ and activating certain transcription factors (18). Further investigation was performed by the present study to determine whether V8-induced apoptosis was associated with the 

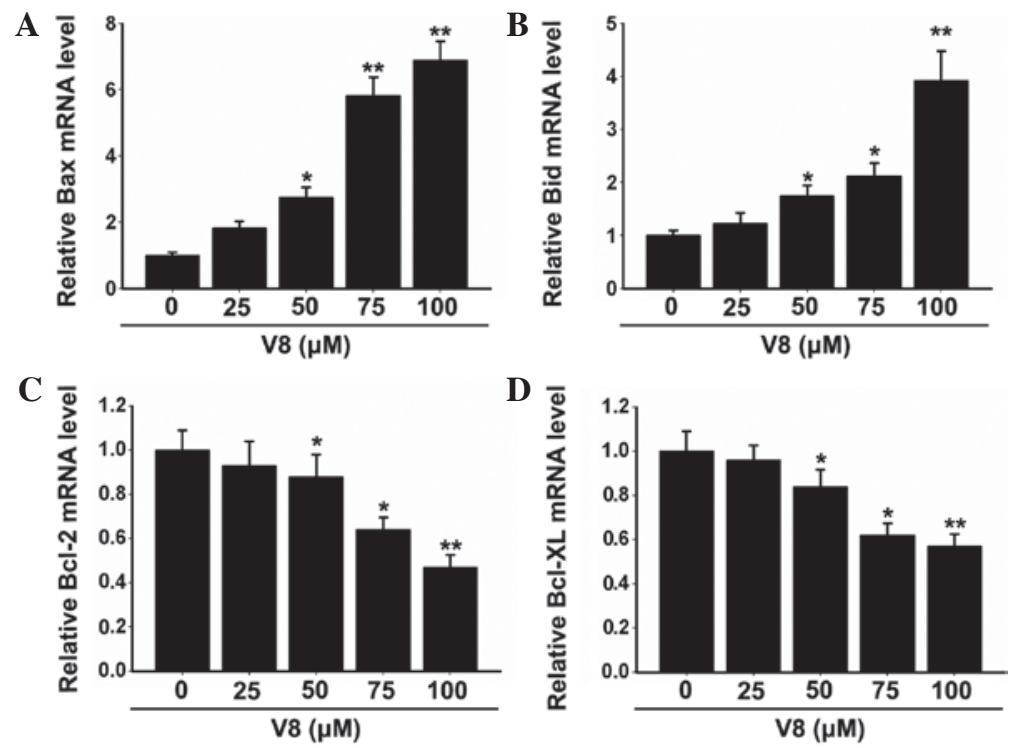

Figure 3. Expression of apoptosis-associated factors in human multiple myeloma RPMI 8226 cells following treatment with V8. The mRNA expression of (A) Bax, (B) Bid, (C) Bcl-2 and (D) Bcl-XL in RPMI 8226 cells following treatment with various concentrations of V8 for $48 \mathrm{~h}$ was detected by quantitative polymerase chain reaction. The fold change was calculated using the $2^{-\Delta \Delta \mathrm{Cq}}$ method vs. glyceraldehyde 3-phosphate dehydrogenase. Data are presented as the mean \pm standard error of the mean of three independent experiments. ${ }^{*} \mathrm{P}<0.05,{ }^{* *} \mathrm{P}<0.01$ vs. untreated cells. V8, 7-\{4-[Bis-(2-hydroxyethyl)-amino]-butoxy\}-5-hydroxy-8-methoxy-2-phenylchromen-4-one; Bcl, B-cell lymphoma; Bax, Bcl-2-like protein 4; Bid, BH3 interacting domain death agonist; Bcl-XL, Bcl-extra large.

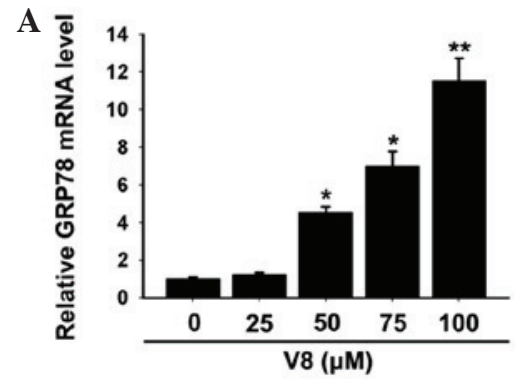

D

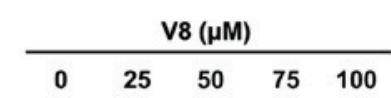

cleaved caspase-12

$\beta$-actin

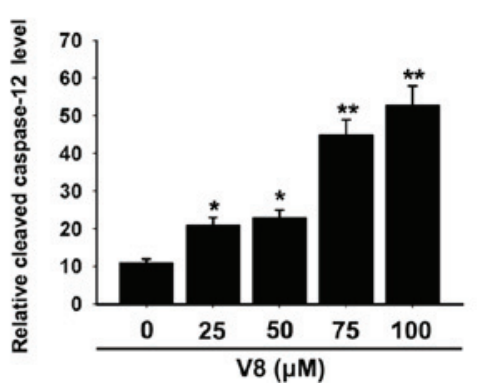

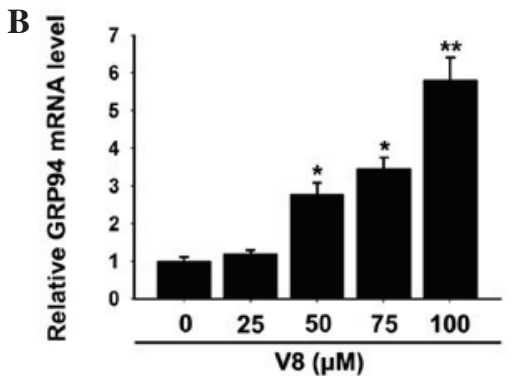

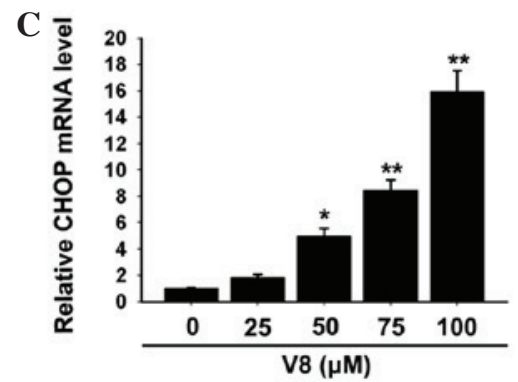

$\mathbf{E}$
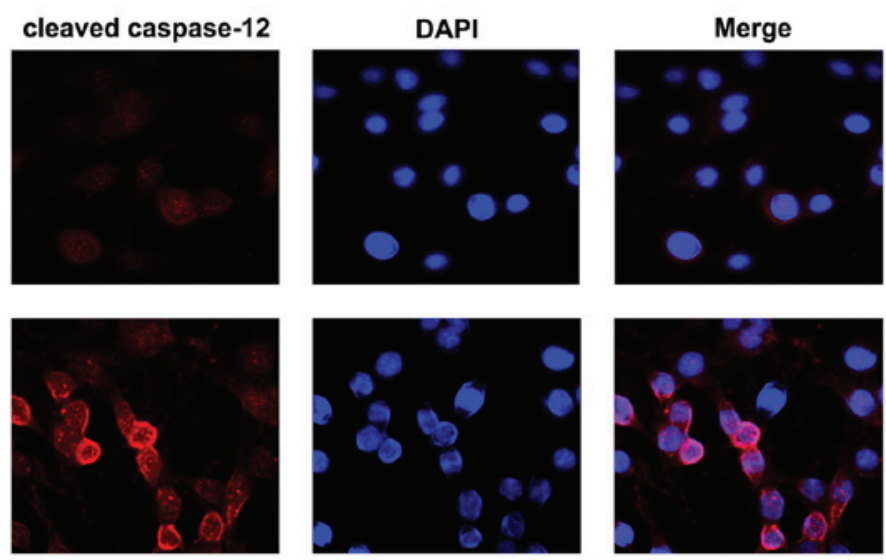

Figure 4. ER stress response was activated by V8. Relative mRNA expression of (A) GRP78, (B) GRP94 and (C) CHOP in human multiple myeloma RPMI 8226 cells was detected following treatment with various concentrations of V8 (25,50,75 and $100 \mu \mathrm{M})$ for $48 \mathrm{~h}$ by quantitative polymerase chain reaction. (D) Alteration in the expression of cleaved caspase-12 following V8 treatment was determined by western blotting. The bar chart demonstrates the level of cleaved caspase-12 relative to glyceraldehyde 3-phosphate dehydrogenase by densitometry. (E) Cleaved caspase-12 (red) and DAPI (blue) immunofluorescence double stain was performed to detect the level and distribution of cleaved caspase-12 in RPMI 8226 cells treated with $75 \mu \mathrm{MV} 8$. Scale bar, $20 \mu \mathrm{m}$. Data are presented as the mean \pm standard error of the mean of three independent experiments. ${ }^{*} \mathrm{P}<0.05,{ }^{* *} \mathrm{P}<0.01$ vs. untreated cells. V8,7-\{4-[Bis-(2-hydroxyethyl)-amino]-butoxy\}-5-hydroxy-8-methoxy-2-phenylchromen-4-one; GRP, glucose-regulated protein; CHOP, C/EBP homologous protein; DAPI, 4',6-diamidino-2-phenylindole; nor, untreated RPMI 8226 cells.

UPR and PERK-eIF2 $\alpha$-ATF4 signaling pathway. Notably, phosphorylation of PERK and eIF2 $\alpha$ was increased by V8 treatment (Fig. 5A-C; $\mathrm{P}<0.05$ ). In addition, the expression of ATF4 was promoted by V8 treatment (Fig. 5A and D; $\mathrm{P}<0.05$ ). 
A
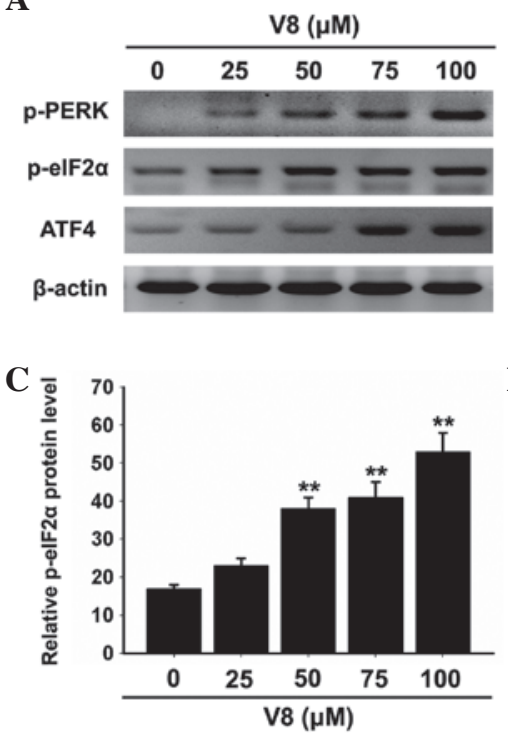

B
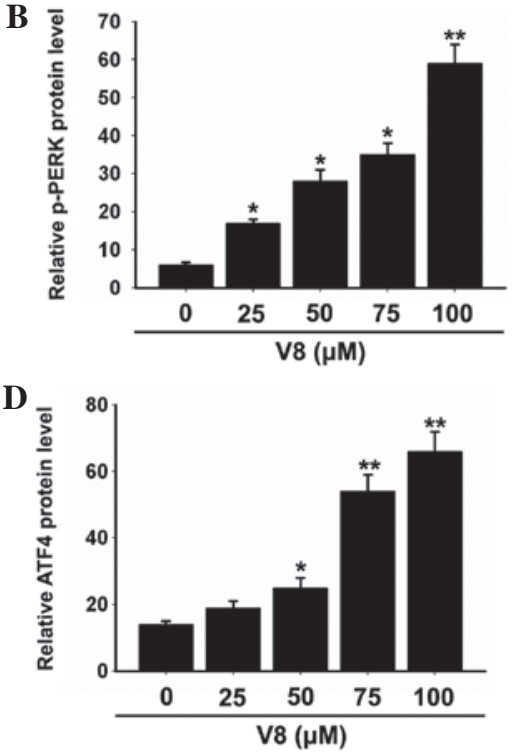

Figure 5. PERK-eIF2 $\alpha$-ATF4 pathway is involved in V8-induced apoptosis of human multiple myeloma RPMI 8226 cells. (A) Alterations of p-PERK, p-eIF2 $\alpha$ and ATF-4 expression at the protein level following V8 treatment was determined by western blotting. (B-D) Ratio of (B) p-PERK, (C) p-eIF2 $\alpha$ and (D) ATF-4 relative to $\beta$-actin in RPMI 8226 cells for various concentrations of V8 by densitometry. Data are presented as the mean \pm standard error of the mean of three independent experiments. ${ }^{*} \mathrm{P}<0.05,{ }^{* *} \mathrm{P}<0.01$ vs. untreated cells. V8, 7-\{4-[Bis-(2-hydroxyethyl)-amino]-butoxy\}-5-hydroxy-8-methoxy-2-phenylchromen-4-one; p-PERK, phosphorylated protein kinase RNA-like endoplasmic reticulum kinase; p-eIF2 $\alpha$, phosphorylated eukaryotic initiation factor $2 \alpha$; ATF4, activating transcription factor 4 .

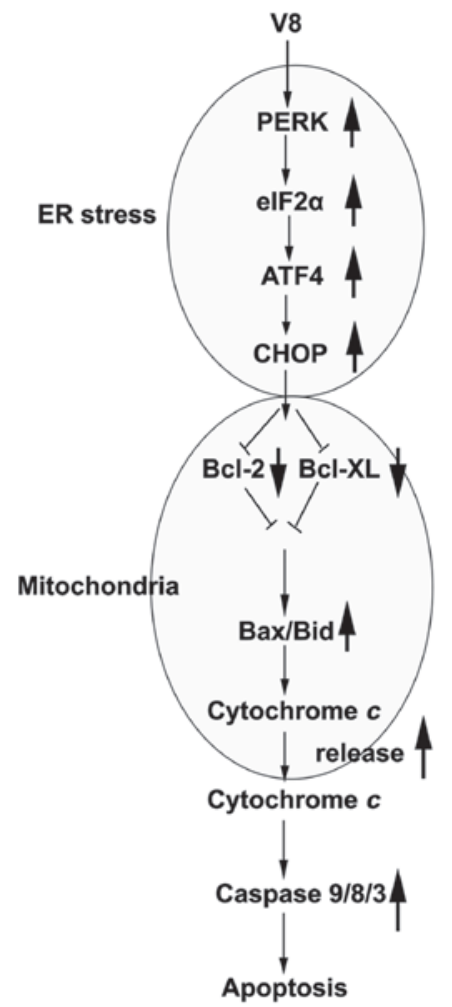

Figure 6. Proposed mechanism by which V8 induces apoptotic cell death in human multiple myeloma RPMI 8226 cells. V8 activates the PERKeIF2 $\alpha$-ATF4-CHOP axis of ER stress signaling. Increasing the expression of PERK, eIF2 $\alpha$, ATF 4 and CHOP promotes the expression of Bcl-2 and Bcl-XLin mitochondria. Activation of the mitochondrial apoptosis pathway induces the upregulation of $\mathrm{Bax} / \mathrm{Bid}$, and the release of cytochrome $c$ into cytoplasm. This increasestheactivity of caspase- $9 / 8 / 3$ and leadstocellapoptosis. V8,8,7- $\{4-[\mathrm{Bis}$ -(2-hydroxyethyl)-aminol-butoxy\}-5-hydroxy-8-methoxy-2-phenylchromen4-one; ER, endoplasmic reticulum; PERK, protein kinase RNA-like endoplasmic reticulum kinase; eIF2 $\alpha$, eukaryotic initiation factor $2 \alpha$; ATF4, activating transcription factor 4; $\mathrm{CHOP}, \mathrm{C} / \mathrm{EBP}$ homologous protein; $\mathrm{Bcl}$, B-cell lymphoma; Bcl-XL, Bcl-extra large; Bax, Bcl-2-like protein 4; Bid, $\mathrm{BH} 3$ interacting domain death agonist.
The activation of the PERK-eIF2 $\alpha$-ATF4 signaling pathway was consistent with the increasing levels of GPR78, GPR94 and CHOP. These results suggest that the PERK-eIF2 $\alpha$-ATF4 signaling pathway and ER stress response are involved in V8 induced apoptosis of RPMI 8226 cells.

\section{Discussion}

Traditional Chinese medicines, including Curcuma longa, $C$. phaeocaulis and $C$. wenyujin, which are well known herbal medicines, have been proposed to have cytotoxic and antitumor properties with lower toxicity and fewer side effects compared with traditional chemotherapeutic agents (19). Due to the extensive anti-inflammatory, anti-angiogenesis and anti-microbial biological activity reported for the chemical constituents of certain plants, including in Alzheimer's disease (20), studies have been performed to understand the function and mechanism of flavonoids in cancer cells $(21,22)$. Various chemical constituents of Chinese medicine have been considered as a novel source of anti-cancer drugs; however, the molecular mechanisms of their actions remain largely unknown. The present study demonstrated that the novel compound V8, derived from natural wogonin, induces apoptosis and ER stress in human MM RPMI 8226 cells in a dose- and time-dependent manner.

Apoptosis has been widely accepted as an important mechanism that contributes to cell death and survival, and is a major treatment modality to kill cancer cells (23). It is well-established that caspase-3, a member of the caspase family enzymes, is the key effector caspase that executes apoptosis, and is activated by initiators, including caspase- 8 and -9 , through mitochondrial-mediated pathways in response to various stimulation (10). In the current study, the activity of caspase-3, -8 and -9 was greatly enhanced by V8 treatment, suggesting that V8-induced apoptosis is associated with the activation of the caspase cascade. 
To understand the molecular basis of V8-induced apoptosis in RPMI 8226 cells, leakage of cyto $c$ from mitochondria to the cytosol, which is regarded as a preceding event for the activation of caspase cascades (11), was evaluated. The present study demonstrated that the level of cyto $c$ was increased in mitochondria and decreased in the cytoplasm indicating that the release of mitochondrial cyto $c$ was promoted by V8 treatment. Cyto $c$ release is mediated and tightly regulated by the Bcl-2 family of proteins, which consists of pro- and anti-apoptotic proteins (24). In previous studies, dimerization of Bax and Bcl-2 homologous antagonist/killer (Bak) induced cyto $c$ release from mitochondria, while anti-apoptotic Bcl-2 family members functioned as dominant negative inhibitors by binding and inhibiting Bax and Bak $(25,26)$. In the present study, the upregulation of Bax and Bid and the downregulation of Bcl-2 and Bcl-XL were observed following V8 treatment, which induced clear cyto $c$ leakage to the cytosol in RPMI 8226 cells.

According to the present data, it is clearly conceivable that the alteration of Bcl-2 family members in RPMI 8226 cells initiates the mitochondrial-initiated events leading to cyto $c$ release and activation of the caspase cascade. Previous studies have demonstrated that activation of caspase- 12 occurs prior to the activation of executioner caspase- 3 in the apoptosis of various cells associated with ER-stress $(17,18,27)$. Consistent with the results of previous studies (11), the protein level of cleaved caspase-12 was increased following the same concentration of V8 stimulation in the present study. Caspase-12 is localized to the cytosolic interface of the ER once it is cleaved and activated, which renders it vulnerable to ER stress, leading to further activation of the caspase cascade $(28,29)$. Therefore, the present study considered the possibility that ER stress may be activated by V8.

The ER has numerous general functions, including the folding of protein molecules, transport of synthesized proteins in vesicles to the Golgi apparatus, posttranslational modifications, lipid and steroid synthesis and calcium signaling. Various conditions, such as ischemia, hypoxia, heat shock, gene mutation and elevated protein synthesis, may impair ER function and result in ER stress, a state in which protein folding slows, leading to an increase in unfolded proteins. This type of stress is characterized by the upregulation of ER chaperones, including GRP78 and GRP94. It is widely known that excessive and prolonged ER stress triggers the cell apoptotic signaling pathway (30). The current study demonstrated that V8 treatment resulted in upregulation of the ER stress response proteins GRP78, GRP94 and CHOP in RPMI 8226 cells. The transcription factor CHOP is also activated in ER stress and causes downregulation of the anti-apoptotic mitochondrial protein Bcl-2 (31). In addition, phosphorylation of PERK and eIF2 $\alpha$ were enhanced, and the expression of ATF4 was clearly induced by V8 treatment in the present study. In response to ER stress, PERK and other protein kinases initiate the UPR, which is tightly associated with the regulation of programmed cell death (32). A study by Rouschop et al (33) indicated that the UPR of the PERK-eIF2 $\alpha$-ATF4 pathway was a potent stimulator of autophagy and apoptosis in response to ER. The present results suggest that V8-induced apoptosis is mediated by ER stress associated with the PERK-eIF2 $\alpha$-ATF4 cascade, which further induced apoptotic signals downstream.
In conclusion, on the basis of the present results, the present study considers the possibility that V8 activates the PERK-eIF2 $\alpha$-ATF4-CHOP axis of ER stress signaling, and that increasing the expression of PERK, eIF $2 \alpha$, ATF4 and $\mathrm{CHOP}$ promotes the expression of Bcl-2 and Bcl-XL in mitochondria. Consequently, the activation of the mitochondrial apoptosis pathway induces the upregulation of $\mathrm{Bax} / \mathrm{Bid}$, which affects mitochondrial outer membrane permeabilization and promotes the release of cyto $c$ into cytoplasm. This increases the activity of caspase- $9,-8$ and -3 , ultimately leading to cell apoptosis (Fig. 6). Overall, V8-induced apoptosis and ER stress in human MM RPMI 8226 cells is associated with the PERK-eIF2 $\alpha$-ATF4 signaling pathway. This suggests that the small molecule V8 may target the ER stress response, and therefore may possess great pharmaceutical value to improve the treatment efficacy of MM.

\section{References}

1. Palumbo A and Anderson K: Multiple myeloma. N Engl J Med 364: 1046-1060, 2011.

2. Sergentanis TN, Kastritis E, Terpos E, Dimopoulos MA and Psaltopoulou T: Cytogenetics and survival of multiple myeloma: Isolated and combined effects. Clin Lymphoma Myeloma Leuk 16: 335-340, 2016.

3. Kumar SK, Rajkumar SV, Dispenzieri A, Lacy MQ, Hayman SR, Buadi FK, Zeldenrust SR, Dingli D, Russell SJ, Lust JA, et al: Improved survival in multiple myeloma and the impact of novel therapies. Blood 111: 2516-2520, 2008.

4. Colson K: Treatment-related symptom management in patients with multiple myeloma: A review. Support Care Cancer 23: 1431-1445, 2015.

5. Aslan E, Guler C and Adem S: In vitro effects of some flavonoids and phenolic acids on human pyruvate kinase isoenzyme M2. J Enzyme Inhib Med Chem 31: 314-317, 2016.

6. Khan R, Saif AQ, Quradha MM, Ali J, Rauf A and Khan A: Antioxidant, antimicrobial and urease inhibiting activities of methanolic extracts from Cyphostemma digitatum stem and roots. Nat Prod Res 30: 486-488, 2016.

7. Pirouzpanah MB, Sabzichi M, Pirouzpanah S, Chavoshi H and Samadi N: Silibilin-induces apoptosis in breast cancer cells by modulating p53, p21, Bak and Bcl-xl Pathways. Asian Pac J Cancer Prev 16: 2087-2092, 2015.

8. Wang Y, Han A, Chen E, Singh RK, Chichester CO, Moore RG, Singh AP and Vorsa N: The cranberry flavonoids PAC DP-9 and quercetin aglycone induce cytotoxicity and cell cycle arrest and increase cisplatin sensitivity in ovarian cancer cells. Int J Oncol 46: 1924-1934, 2015.

9. Hung JY, Chang WA, Tsai YM, Hsu YL, Chiang HH, Chou SH, Huang MS and Kuo PL: Tricetin, a dietary flavonoid, suppresses benzo(a)pyrene-induced human non-small cell lung cancer bone metastasis. Int J Oncol 46: 1985-1993, 2015.

10. Ge W, Yin Q and Xian H: Wogonin induced mitochondrial dysfunction and endoplasmic reticulum stress in human malignant neuroblastoma cells via IRE1 $\alpha$-dependent pathway. J Mol Neurosci 56: 652-662, 2015.

11. Falank C, Fairfield H and Reagan MR: Signaling interplay between bone marrow adipose tissue and multiple myeloma cells. Front Endocrinol (Lausanne) 7: 67, 2016.

12. Rengarajan T and Yaacob NS: The flavonoid fisetin as an anticancer agent targeting the growth signaling pathways. Eur J Pharmacol: Jul 1, 2016 (Epub ahead of print).

13. Zhang Y, Zhao L, Li X, Wang Y, Yao J, Wang H, Li F, Li Z and Guo Q: V8, a newly synthetic flavonoid, induces apoptosis through ROS-mediated ER stress pathway in hepatocellular carcinoma. Arch Toxicol 88: 97-107, 2014.

14. HeA,JiR,ShaoJ,HeC,JinMandXuY:TLR4-MyD88-TRAF6-TAK1 complex-mediated NF-kappaB activation contribute to the antiinflammatory effect of V8 in LPS-induced human cervical cancer SiHa cells. Inflammation 39: 172-181, 2016.

15. Liu J, Gratz J, Amour C, Nshama R, Walongo T, Maro A, Mduma E, Platts-Mills J, Boisen N, Nataro J, et al: Optimization of quantitative PCR methods for enteropathogen detection. PLoS One 11: e0158199, 2016. 
16. Bhola PD and Letai A: Mitochondria - Judges and executioners of cell death sentences. Molecular cell 61: 695-704, 2016.

17. Bittremieux M, Parys JB, Pinton P and Bultynck G: ER functions of oncogenes and tumor suppressors: Modulators of intracellular $\mathrm{Ca}(2+)$ signaling. Biochim Biophys Acta 1863: 1364-1378, 2016.

18. Lagace TA and Ridgway ND: The role of phospholipids in the biological activity and structure of the endoplasmic reticulum. Biochim Biophys Acta 1833: 2499-2510, 2013.

19. Li-Weber M: New therapeutic aspects of flavones: The anticancer properties of Scutellaria and its main active constituents Wogonin, Baicalein and Baicalin. Cancer Treat Rev 35: 57-68, 2009.

20. Volgyi K, Juhasz G, Kovacs Z and Penke B: Dysfunction of endoplasmic reticulum (ER) and mitochondria (MT) in Alzheimer's disease: The role of the ER-MT cross-talk. Curr Alzheimer Res 12: 655-672, 2015.

21. Lall RK, Adhami VM and Mukhtar H: Dietary flavonoid fisetin for cancer prevention and treatment. Mol Nutr Food Res 60: 1396-1405, 2016

22. Devi KP, Rajavel T, Habtemariam S, Nabavi SF and Nabavi SM: Molecular mechanisms underlying anticancer effects of myricetin. Life Sci 142: 19-25, 2015.

23. Kelloff GJ, Crowell JA, Steele VE, Lubet RA, Malone WA, Boone CW, Kopelovich L, Hawk ET, Lieberman R, Lawrence JA, et al: Progress in cancer chemoprevention: Development of diet-derived chemopreventive agents. J Nutr 130 (Suppl 2S): S467-S471, 2000.

24. Kurokawa M and Kornbluth S: Caspases and kinases in a death grip. Cell 138: 838-854, 2009.
25. Suen DF, Norris KL and Youle RJ: Mitochondrial dynamics and apoptosis. Genes Dev 22: 1577-1590, 2008.

26. Martinou JC and Youle RJ: Mitochondria in apoptosis: Bcl-2 family members and mitochondrial dynamics. Dev Cell 21: 92-101, 2011.

27. Moorwood C and Barton ER: Caspase-12 ablation preserves muscle function in the $\mathrm{mdx}$ mouse. Hum Mol Genet 23: 5325-5341, 2014

28. Liu H, Wang Z and Nowicki MJ: Caspase-12 mediates carbon tetrachloride-induced hepatocyte apoptosis in mice. World J Gastroenterol 20: 18189-18198, 2014.

29. Nakagawa T, Zhu H, Morishima N, Li E, Xu J, Yankner BA and Yuan J: Caspase-12 mediates endoplasmic-reticulum-specific apoptosis and cytotoxicity by amyloid-beta. Nature 403: 98-103, 2000.

30. Sun Y, Zhang T, Li L and Wang J: Induction of apoptosis by hypertension via endoplasmic reticulum stress. Kidney Blood Press Res 40: 41-51, 2015.

31. Zhang YS, Shen Q and Li J: Traditional Chinese medicine targeting apoptotic mechanisms for esophageal cancer therapy. Acta Pharmacol Sin 37: 295-302, 2016.

32. Jiang Q, Li F, Shi K, Wu P, An J, Yang Y and Xu C: Involvement of p38 in signal switching from autophagy to apoptosis via the PERK/eIF2 $\alpha /$ ATF4 axis in selenite-treated NB4 cells. Cell Death Dis 5: e1270, 2014.

33. Rouschop KM, van den Beucken T, Dubois L, Niessen H, Bussink J, Savelkouls K, Keulers T, Mujcic H, Landuyt W, Voncken JW, et al: The unfolded protein response protects human tumor cells during hypoxia through regulation of the autophagy genes MAP1LC3B and ATG5. J Clin Invest 120: 127-141, 2010. 